\title{
Studies on Isolation, Characterization and in-vitro Screening of Plant Growth Promoting Rhizobacteria from Rhizospheric Soil of Chrysanthemum (Dendranthema grandiflora Tzvelev.)
}

\author{
Praveen Ranadev*, K. Nagaraju, R. Muthuraju and R. Vasantha Kumari \\ Department of Agricultural Microbiology, University of Agricultural Sciences, GKVK, \\ Bengaluru, Karnataka, India \\ *Corresponding author
}

\begin{abstract}
A B S T R A C T
A total of forty rhizosphere soil samples were collected from different locations of Bengaluru urban district, Karnataka and used for isolation of PGPRs by standard dilution

Keywords

Azotobacter,

Bacillus,

Pseudomonas,

Chrysanthemum,

PGPR

Article Info

Accepted:

07 May 2019

Available Online:

10 June 2019 plate method. Seventy bacterial isolates were selected, purified and were examined for morphological characters like colony morphology, cell shape, Gram reaction. Isolates were diverse in their morphology ranging from dull to milky white, rough to smooth margins, viscous, small to large, non-spreading and spreading. Among them twenty seven and seventeen isolates were identified as $\mathrm{N}_{2}$ fixers and as Phosphate solubilizers by initial screening respectively. Further, the selected PGPR isolates were biochemically characterized and identified as Azotobacter spp. Bacillus spp. and Pseudomonas sp. The isolates screened qualitatively and quantitatively for plant growth promotion activities like $\mathrm{N}_{2}$ fixation, phosphate solubalization and growth hormone production (IAA). The amount of nitrogen fixed in Waksman No.77 nitrogen free broth ranged from 4.52 to $19.33 \mathrm{mg}$ per $\mathrm{g}$ of $\mathrm{C}$ utilized and highest fixed by isolate PGPR-24. Phosphate solubalization efficiency and phosphate solubilization activity in Pikovskaya's medium was ranged from $102.8 \%$ to $168.7 \%$ and $8.56 \%$ to $14.32 \%$ respectively and more phosphate solubilization activity showed by isolatePGPR-9. Indole acetic activity (IAA) production ranged between 4.54 to $25.12 \mu \mathrm{g}$ per $\mathrm{ml}$.
\end{abstract}

\section{Introduction}

Plant growth-promoting rhizobacteria (PGPR) are naturally occurring soil bacteria that aggressively colonize plant roots and benefit plants. They are a heterogeneous group of bacteria that can be found in the rhizosphere, on rhizoplane and in association with roots, enhancing the growth of the plant either directly and/or indirectly (Glick, 1995) through effective mobilization of major plant nutrients like Nitrogen, Phosphorus, Potash and other minor nutrients needed by the crop. These beneficial microorganisms are also known to secret plant growth promoting substance like IAA, GA, cytokinines and vitamins for the improvement of crop growth, yields and for quality produce. They not only 
promote plant growth but also help in sustainable agricultural development and protecting the environment (Das et al., 2013). It is well established that only 1 to 2 per cent of bacteria promote plant growth in the rhizosphere (Antoun and Kloepper, 2001).

The most predominant rhizosphere colonizing bacteria belong to genus Bacillus, Pseudomonas, Azotobacter etc., because of their rapid growth rate in soils having varying amount of organic matter, tolerance to different agricultural pesticides those are generally used in seed dressing (Vijaypal, 1998). The present investigation was conducted for isolation, characterization and screening of efficient strains of PGPR isolates.

\section{Materials and Methods}

The present investigation was carried out at the Department of Agricultural Microbiology, University of Agricultural Sciences, Bengaluru for screening of efficient PGPR isolates (free living $\mathrm{N}_{2}$ fixers and phosphate solubilizing bacteria) isolated from rhizospheric soil of chrysanthemum crop grown in various parts of Anekal taluk, Bengaluru urban district, Karnataka.

\section{Isolation and characterization}

All the PGPR isolates Azotobacter, Bacillus and Pseudomonas were isolated from the rhizosphere soil samples of chrysanthemum collected from various parts of Anekal taluk, Bengaluru urban district, Karnataka using standard serial dilution method. The Azotobacter isolates were isolated by using Waksman No.77 medium, Mannitol: $20.0 \mathrm{~g}$, $\mathrm{K}_{2} \mathrm{HPO}_{4}: 0.2 \mathrm{~g}, \mathrm{MgSO}_{4} .7 \mathrm{H}_{2} \mathrm{O}: 0.2 \mathrm{~g}, \mathrm{NaCl}$ : $0.2 \mathrm{~g}, \mathrm{~K}_{2} \mathrm{SO}_{4}: 0.1 \mathrm{~g}, \mathrm{CaCO}_{3}: 5.0 \mathrm{~g}$, Agar: 15.0 $\mathrm{g}$ per lit of distilled water and Phosphate solubilizers were isolated by using Pikovskaya's agar medium contented
Glucose: $10 \mathrm{~g}, \mathrm{Ca}_{3}\left(\mathrm{PO}_{4}\right)_{2}: 5 \mathrm{~g},\left(\mathrm{NH}_{4}\right)_{2} \mathrm{SO}_{4}$ : $0.5 \mathrm{~g}, \mathrm{KCl}: 0.2 \mathrm{~g}, \mathrm{MgSO}_{4}: 0.1, \mathrm{MnSO}_{4}$ and $\mathrm{FeSO}_{4}$ : Trace, Yeast extract: $0.5 \mathrm{~g}, \mathrm{NaCl}: 0.1$ g, Agar: $20 \mathrm{~g}$ for lit of distilled water (Pikovskaya, 1948). The purified isolates were transferred to the respective slants of the same medium and stored for further studies.

The PGPR isolates were characterized by their cultural conditions, morphological and biochemical characteristics (Indole production, Methyl red test, Voges-Proskauer test, Citrate utilization test, Catalase test, Oxidase test, Starch hydrolysis, Gelatine liquefaction, $\mathrm{H}_{2} \mathrm{~S}$ production, Acid and gas production, Casein hydrolysis, Urease test and Utilization of different carbon sources) using standard methods (Cappuccino and Sherman, 1992).

\section{In vitro assessment of PGP traits of rhizobacterial isolates}

\section{Screening of isolates for quantitative} estimation of in vitro nitrogen fixation

All the free living nitrogen fixing isolates were examined for quantitative Nitrogenfixation as per standard procedures. $100 \mathrm{ml}$ of the medium (Waksman No. 77) was dispensed in $250 \mathrm{ml}$ conical flasks and autoclaved. One $\mathrm{ml}$ of $24 \mathrm{hr}$ old culture was inoculated to each flask. The flasks were incubated at $37^{\circ} \mathrm{C}$ for seven days. After seven days of incubation, the culture was homogenized and $10 \mathrm{ml}$ was digested with 5 ml of concentrated $\mathrm{H}_{2} \mathrm{SO}_{4}$ along with $0.2 \mathrm{~g}$ digestion catalyst mixture $\mathrm{K}_{2} \mathrm{SO}_{4}: \mathrm{CuSO}_{4}$ : Selenium (100:10:1). After cooling, volume was made up to $10 \mathrm{ml}$ with distilled water. Later, $10 \mathrm{ml}$ of aliquot was transferred to microkjeldhal distillation unit, for which 20 $\mathrm{ml}$ of 40 per cent $\mathrm{NaOH}$ was added and distilled. Ammonia evolved was trapped in 4 per cent boric acid mixed indicator (Bromocresol green $0.066 \mathrm{~g}$ and methyl red 
$0.033 \mathrm{~g}$ in $100 \mathrm{ml}$ methanol) till the solution turned from pink to green. It was titrated against $0.05 \quad \mathrm{~N} \mathrm{H}_{2} \mathrm{SO}_{4}$ and total nitrogen content of the culture was determined and results were expressed as $\mathrm{mg} \mathrm{N}$ fixed per $\mathrm{g}$ of malate.

Nitrogen fixed $(\%)=$

Titre value $\times 0.014 \times \mathrm{N}$ of $\mathrm{H}_{2} \mathrm{SO}_{4} \times$ vol. made

Volume of sample used

Screening of PSB isolates for in vitro phosphate solubilization

Phosphate solubilizing ability of each PSB isolate was determined by measuring the zone of $\mathrm{PO}_{4}{ }^{-}$solubilization on the Pikovskaya's agar medium and by estimating per cent $\mathrm{Pi}$ released in Pikovskaya's broth medium.

\section{Qualitative estimation [Phosphate solubilization efficiency]}

For determining zone of Phosphate solubilization, about $20 \mathrm{ml}$ of Pikovskaya's agar medium was added into the sterilized petriplates, after solidification $10 \mu \mathrm{l}$ broth culture was spot inoculated on the plates. The plates were incubated at $28 \pm 2{ }^{\circ} \mathrm{C}$ for about 3-5 days and solubilization zone was observed around the colony. Phosphate solubilization efficiency was calculated by using the following formula.

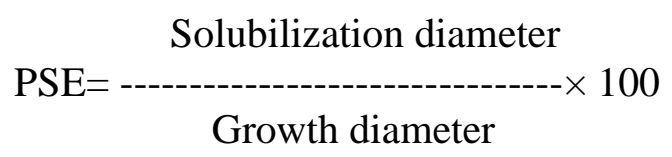

\section{Quantitative estimation of Pi released from tricalcium phosphate}

The isolates showing zone of solubilization on Pikovskaya's agar were further examined for their ability to release Pi from TCP in broth medium. One $\mathrm{ml}$ of overnight culture was inoculated to $50 \mathrm{ml}$ of Pikovskaya's broth (Pikovaskya, 1948). The inoculated flask was incubated for two weeks at $28 \pm 2{ }^{\circ} \mathrm{C}$. The amount of $\mathrm{Pi}$ released in the broth was estimated at 5 and 10 days of incubation from triplicate flask at each stage in comparison with a set of uninoculated controls. The broth cultures were centrifuged at $10,000 \mathrm{rpm}$ for 10 minutes in a centrifuge to separate the supernatant from the cell growth and insoluble phosphate. The available Phosphorus content in the supernatant was estimated by phosphomolybdic blue color method (Jackson, 1973).

\section{Quantitative test for IAA production by spectrophotometric method}

All the PGPR isolates were further examined for the amount of IAA production. The IAA production by isolates was determined by following the method of Ivanova et al., (2001) under in vitro condition.

\section{Results and Discussion}

\section{Isolation and morphological characterization of PGPRs}

On the basis of cultural, morphological and biochemical characteristics out of 70 bacterial isolates 44 bacterial isolates were showed plant growth promotion activity under in vitro condition. 27 were grouped as nitrogen fixers and 17 isolates were grouped as Phosphate solubilizers as described in Bergey's Manual of Determinative Bacteriology (Holt et al., 1994). All the $\mathrm{N}_{2}$ fixing isolates were Gram -ve, rod, cocci, dull white or Milky white, round, non-spreading, smooth, raised, opaque, viscid colony were identified as Azotobacter spp. Whereas in PSB isolates some are Gram + , rod shaped cell, Large, smooth, soft, glistening, round, convex, entire, nonspreading, dense, creamy white to yellow colony were identified as Bacillus spp. and some are Gram -, rod shaped, Yellowish 
green, round, non-spreading, glistening, convex, opaque, viscid colony were identified as Pseudomonas sp. (Table 1).

\section{Biochemical characterization of PGPR isolates}

Different biochemical tests were conducted to identify nitrogen fixers and phosphate solubilizers. Most of the nitrogen fixing isolates was shown positive result for indole production, methyl red test, oxidase test, gelatin liquification, ammonia production, acid production, casein hydrolysis and motility and negative for VP test and gas production. In terms of carbon source utilization most of the isolates shown good growth in medium supplemented with mannitol as carbon sources and this isolates were identified as Azotobacter sp. (Table 2).

PSB isolates were shown positive result for Catalase test, acid production, casein hydrolysis and motility whereas negative for Oxidase, gas production, $\mathrm{H}_{2} \mathrm{~S}$ production and most of the isolates shown variations in results (Table 3). All the PSB isolates utilize glucose and sucrose as carbon sources but fail to utilize mannitol and were identified as Bacillus spp. and Pseudomonas spp.

\section{Screening of free living $\mathrm{N}_{2}$ - fixing isolates for in vitro nitrogen fixation}

$\mathrm{N}_{2}$ fixation is naturally the first major mechanism of action suggested for the enhancement of plant growth by Azotobacter. In the present investigation all the free living $\mathrm{N}_{2}$ fixing isolates were tested for in-vitro nitrogen fixation, all 27 isolates fixed nitrogen but the quantity of $\mathrm{N}_{2}$ fixation varied with individual isolate ranged from 4.52 to 19.33 $\mathrm{mg}$ per $\mathrm{g}$ of $\mathrm{C}$ utilized and detail was given in Table 4. Highest nitrogen fixation was observed in isolate PGPR-24 (19.33 mg per g $C$ source) which was superior over all the isolates, followed by PGPR-45 (17.78 mg per $\mathrm{g}$ of C source). Kanimozhi et al., (2010), the nitrogen fixation ability of the 30 isolates of Azotobacter from paddy rhizosphere soil of Thanjavur district by using micro kjeldhal method. Among the 30 isolates tested, 28 isolates were able to fix nitrogen. The range of nitrogen fixing ability was from 3.3 to 15.6 $\mathrm{mg} \mathrm{N} / \mathrm{g}$ malate. Similar work has done by Goswmy (1976) and Kizilkaya (2009).

\section{Screening of PSB isolates for in vitro phosphate solubilization}

\section{Phosphate solubilization efficiency (PSE)}

Data on qualitative analysis of the PSB isolates for $\mathrm{P}$ solubilization is presented in Table 5. Out of 17 isolates examined for their ability to solubilize TCP in Pikovskaya's agar medium supplemented with TCP as insoluble $\mathrm{P}$ source. all the isolates showed zone of solubilization, in that isolate PGPR-9 showed the highest Phosphate solubilization (168.7 $\%)$ followed by reference strain $(150.00 \%)$ whereas lowest solubilization was found in PGPR-42 (106.8\%) and also this was conformity with the findings of Sharma et al., 2007 and Guar et al, (1973), they also reported that Bacillus megaterium var. phosphaticum recorded maximum percentage of phosphate solubilization.

\section{Quantitative solubilization of phosphate} (Per cent Pi released)

Per cent Pi released by PSB isolates at different intervals viz., $5^{\text {th }}$ and $10^{\text {th }}$ day of incubation (Table 5). The highest Pi released was observed by isolate PGPR-9 at $5^{\text {th }}$ day of incubation $(7.85 \%)$ and at $10^{\text {th }}$ day of incubation (14.32\%) compared to all PSB isolates and isolate PGPR-57 released considerable amount of Phosphate at $5^{\text {th }}(6.57$ $\%)$ and $10^{\text {th }}(13.13 \%)$ day of incubation respectively. 
Table.1 Morphological characterization and identification of plant growth promotion activity of bacterial isolates

\begin{tabular}{|c|c|c|c|c|c|c|}
\hline \multirow[t]{2}{*}{ Sl. No } & \multirow[t]{2}{*}{ Isolate code } & \multicolumn{5}{|c|}{ Morphological characters } \\
\hline & & Colony morphology & Cell shape & $\begin{array}{l}\text { Gram } \\
\text { reaction }\end{array}$ & $\begin{array}{l}\text { Growth on } \mathrm{N} \\
\text { free medium }\end{array}$ & $\begin{array}{l}\text { Solubilize } \\
\mathrm{PO}_{4}^{-}\end{array}$ \\
\hline 1 & PGPR-1 & $\begin{array}{l}\text { Large, smooth, soft, glistening, round, convex, entire, non-spreading, dense, } \\
\text { creamy white to yellow. Variations: Rough, }\end{array}$ & Rod & $+\mathrm{ve}$ & - & ++ \\
\hline 2 & PGPR-3 & $\begin{array}{l}\text { Rough, opaque, dull, spreading, off white. Variations: Smooth to slimy, soft, thin, } \\
\text { translucent, dendroid. Yellow, orange or brown. }\end{array}$ & Rod & $+\mathrm{ve}$ & - & + \\
\hline 3 & PGPR-4 & Milky white, round, non-spreading, smooth, raised, opaque, viscid colony & Rod & -ve & + & - \\
\hline 4 & PGPR-5 & Dull white, slimy, round, non-spreading, smooth, raised, opaque, viscid colony & Rod & -ve & ++ & - \\
\hline 5 & PGPR-7 & $\begin{array}{l}\text { Yellowish green, round, non-spreading, glistening, convex, opaque, viscid colony } \\
\text { with green pigmentation. }\end{array}$ & Rod & -ve & -- & ++ \\
\hline 6 & PGPR-8 & Off white, irregular, nonspreading, smooth, flat, opaque, viscid colony & Rod & -ve & + & - \\
\hline 7 & PGPR-9 & $\begin{array}{l}\text { Large, smooth, soft, glistening, round, convex, entire, non-spreading, dense, } \\
\text { creamy white to yellow. Variations: }\end{array}$ & Rod & $+\mathrm{ve}$ & -- & +++ \\
\hline 8 & PGPR-10 & Milky white, round, non-spreading, smooth, raised, opaque, viscid colony & Rod & -ve & + & - \\
\hline 9 & PGPR-12 & $\begin{array}{l}\text { Clear white to brownish, round, non-spreading, smooth, raised, opaque, gummy } \\
\text { colony }\end{array}$ & Rod & -ve & ++ & - \\
\hline 10 & PGPR-13 & $\begin{array}{l}\text { Rough, opaque, dull, spreading, off white. Variations: Smooth to slimy, soft, thin, } \\
\text { translucent, dendroid. Yellow, orange or brown. }\end{array}$ & Rod in chains & $+\mathrm{ve}$ & -- & + \\
\hline 11 & PGPR-17 & Clear white, slimy, non-spreading, smooth, raised, opaque, viscid colony & Rod & -ve & + & - \\
\hline 12 & PGPR-18 & Milky white, spherical, non-spreading, smooth, raised, opaque, viscid colony & Rod & -ve & ++ & - \\
\hline 13 & PGPR-19 & $\begin{array}{l}\text { Large, smooth, soft, glistening, round, convex, entire, non-spreading, dense, } \\
\text { creamy white. Variations: Rough, radially ridged, thin edged }\end{array}$ & Rod in chains & $+\mathrm{ve}$ & -- & + \\
\hline 14 & PGPR-21 & Dull white, round, non-spreading, smooth, raised, opaque, mucoid colony & Rod & -ve & ++ & - \\
\hline 15 & PGPR-23 & $\begin{array}{l}\text { Dull white, round, non-spreading, glistening, convex, opaque, viscid colony with } \\
\text { no pigmentation. }\end{array}$ & Rod & -ve & -- & ++ \\
\hline 16 & PGPR-24 & Milky white, round, non-spreading, smooth, raised, opaque, viscid colony & Rod & $-v e$ & +++ & - \\
\hline 17 & PGPR-27 & $\begin{array}{l}\text { Rough, opaque, dull, spreading, off white. Variations: Smooth to slimy, soft, thin, } \\
\text { translucent, dendroid. Yellow, orange or brown. }\end{array}$ & Rod & $+\mathrm{ve}$ & - & ++ \\
\hline 18 & PGPR-28 & Milky white, round, non-spreading, smooth, raised, opaque, viscid colony & Rod & $-\mathrm{ve}$ & ++ & - \\
\hline 19 & PGPR-29 & $\begin{array}{l}\text { Large, smooth, soft, glistening, round, convex, entire, non-spreading, dense, } \\
\text { creamy white to yellow. Variations: Rough, concentrically or radially ridged, thin } \\
\text { edged }\end{array}$ & Rod & $+\mathrm{ve}$ & -- & + \\
\hline 20 & PGPR-30 & Milky white, round, non-spreading, smooth, raised, & Rod & -ve & ++ & - \\
\hline 21 & PGPR-31 & Dull white, spherical flat, non-spreading, smooth, raised, opaque, gummy colony & Rod & -ve & + & - \\
\hline 22 & PGPR-33 & $\begin{array}{l}\text { Yellowish green, round, non-spreading, glistening, convex, opaque, viscid colony } \\
\text { with green pigmentation. }\end{array}$ & Rod & -ve & - & ++ \\
\hline 23 & PGPR-34 & Milky white, round, non-spreading, smooth, raised, opaque, viscid colony & Rod & -ve & ++ & - \\
\hline 24 & PGPR-37 & Large, smooth, soft, glistening, round, convex, entire, non-spreading, dense, & Rod & + ve & - & ++ \\
\hline
\end{tabular}




\begin{tabular}{|c|c|c|c|c|c|c|}
\hline & & creamy white. Rough, concentrically ridged, thin edged & & & & \\
\hline 25 & PGPR-38 & Milky white, round, non-spreading, smooth, raised, opaque, viscid colony & Rod & -ve & +++ & - \\
\hline 26 & PGPR-41 & $\begin{array}{l}\text { Clear white, round, non-spreading, raised, opaque, viscid colony with light brown } \\
\text { pigmentation. }\end{array}$ & Rod & -ve & ++ & - \\
\hline 27 & PGPR-42 & Yellowish green, round, non-spreading, glistening, convex, & Rod & -ve & -- & ++ \\
\hline 28 & PGPR-43 & Dull white, circular, non-spreading, smooth, raised, opaque, & Rod & -ve & ++ & - \\
\hline 29 & PGPR-45 & Milky white, circular, non-spreading, smooth, raised, opaque, viscid colony. & Rod & -ve & + & - \\
\hline 30 & PGPR-46 & Milky white, round, non-spreading, smooth, raised, opaque, viscid colony. & Rod & -ve & ++ & - \\
\hline 31 & PGPR-47 & $\begin{array}{l}\text { Large, smooth, soft, glistening, round, convex, entire, non-spreading, dense, } \\
\text { creamy white to yellow. Variations: Rough, concentrically or radially ridged, thin } \\
\text { edged }\end{array}$ & Rod in chains & $+\mathrm{ve}$ & -- & + \\
\hline 32 & PGPR-48 & $\begin{array}{l}\text { Large, smooth, soft, glistening, round, convex, entire, non-spreading, dense, } \\
\text { creamy white to yellow. Variations: Rough, concentrically or radially ridged, thin } \\
\text { edged }\end{array}$ & Rod & $+\mathrm{ve}$ & - & ++ \\
\hline 33 & PGPR-49 & white, round, non-spreading, smooth, raised, opaque, viscid colony & Rod & -ve & + & - \\
\hline 34 & PGPR-51 & Milky white, round, non-spreading, smooth, raised, opaque, viscid colony & Rod & -ve & + & - \\
\hline 35 & PGPR-53 & Milky white, round, non-spreading, smooth, raised, opaque, viscid colony & Rod & -ve & ++ & - \\
\hline 36 & PGPR-54 & $\begin{array}{l}\text { Large, smooth, soft, glistening, round, convex, entire, non-spreading, dense, white } \\
\text { to yellow. Variations: Rough, }\end{array}$ & Rod & $+\mathrm{ve}$ & - & + \\
\hline 37 & PGPR-55 & $\begin{array}{l}\text { Rough, opaque, dull, spreading, off white. Variations: Smooth to slimy, soft, thin, } \\
\text { translucent, dendroid. Yellow, orange or brown. }\end{array}$ & Rod & -ve & +++ & - \\
\hline 38 & PGPR-57 & Yellowish green, round, non-spreading, glistening, convex, opaque, viscid colony & Rod & -ve & - & ++ \\
\hline 39 & PGPR-59 & light white, round, non-spreading, smooth, raised, opaque, viscid colony & Rod & -ve & ++ & - \\
\hline 40 & PGPR-61 & Milky white, round, non-spreading, smooth, raised, opaque, viscid colony & Oval & -ve & + & - \\
\hline 41 & PGPR-62 & $\begin{array}{l}\text { Large, smooth, soft, glistening, round, convex, entire, non-spreading, dense, } \\
\text { creamy white to yellow. Variations: Rough, concentrically or radially ridged, thin } \\
\text { edged }\end{array}$ & Rod & $+\mathrm{ve}$ & -- & ++ \\
\hline 42 & PGPR-64 & white, round, non-spreading, smooth, raised, opaque, viscid colony & Rod & -ve & ++ & - \\
\hline 43 & PGPR-65 & Milky white, round, non-spreading, smooth, raised, opaque, viscid colony & Rod & -ve & + & - \\
\hline 44 & PGPR-66 & $\begin{array}{l}\text { Large, smooth, soft, glistening, round, convex, entire, non-spreading, dense, } \\
\text { creamy white to yellow. Variations: Rough, concentrically or radially ridged, thin } \\
\text { edged }\end{array}$ & Rod & $+\mathrm{ve}$ & - & + \\
\hline 45 & PGPR-68 & Milky white, round, non-spreading, smooth, raised, opaque, viscid colony & Coccid & -ve & + & - \\
\hline \multicolumn{2}{|c|}{$\begin{array}{l}+ \text { : Low growth. } \\
\text { +ve: Positive }\end{array}$} & \multicolumn{4}{|c|}{ +++: Good growth } & \\
\hline
\end{tabular}


Table.2 Biochemical characteristics of the free living nitrogen fixers

\begin{tabular}{|c|c|c|c|c|c|c|c|c|c|c|c|c|c|c|c|c|c|c|}
\hline \multirow[b]{2}{*}{ Isolate } & \multicolumn{17}{|c|}{ Biochemical tests } & \multirow[t]{2}{*}{ Probable genera } \\
\hline & 1 & 2 & 3 & 4 & 5 & 6 & 7 & 8 & 9 & 10 & 11 & $12 a$ & $12 b$ & 13 & $14 a$ & $14 b$ & $14 c$ & \\
\hline PGPR-4 & + & + & - & + & + & + & - & + & + & + & + & + & - & + & $3+$ & $2+$ & $4+$ & Azotobacter sp. \\
\hline PGPR-5 & + & + & - & - & + & + & + & + & - & + & + & + & - & + & $3+$ & $2+$ & $3+$ & Azotobacter sp. \\
\hline PGPR-8 & + & + & - & + & + & + & + & + & + & + & + & + & - & + & $4+$ & $3+$ & $4+$ & - \\
\hline PGPR-10 & + & + & - & + & + & + & - & + & + & + & + & + & - & + & $3+$ & $2+$ & $3+$ & Azotobacter sp. \\
\hline PGPR-12 & + & + & - & - & + & + & + & + & - & + & + & + & - & + & $3+$ & $2+$ & $4+$ & Azotobacter sp. \\
\hline PGPR-17 & + & + & - & - & + & + & + & + & - & + & + & + & - & + & $4+$ & $3+$ & $4+$ & Azotobacter sp. \\
\hline PGPR-18 & + & + & - & + & + & + & - & + & + & + & + & + & - & + & $3+$ & $2+$ & $3+$ & Azotobacter sp. \\
\hline PGPR-21 & + & + & - & + & + & + & + & + & + & + & + & + & - & + & $3+$ & $2+$ & $3+$ & Azotobacter sp. \\
\hline PGPR-24 & + & + & - & + & + & + & - & + & + & + & + & + & - & + & $3+$ & $2+$ & $3+$ & Azotobacter sp. \\
\hline PGPR-28 & + & + & - & - & + & + & + & + & - & + & + & + & - & + & $3+$ & $2+$ & $4+$ & Azotobacter sp. \\
\hline PGPR-30 & + & + & - & + & + & + & - & + & + & + & + & + & - & + & $3+$ & $2+$ & $3+$ & Azotobacter sp. \\
\hline PGPR-31 & + & + & - & - & + & + & + & + & - & + & + & + & - & + & $3+$ & $2+$ & $4+$ & Azotobacter sp. \\
\hline PGPR-34 & + & + & - & + & + & + & - & + & + & + & + & + & - & + & $4+$ & $2+$ & $4+$ & - \\
\hline PGPR-38 & + & + & - & + & + & + & - & + & + & + & + & + & - & + & $3+$ & $2+$ & $3+$ & Azotobacter sp. \\
\hline PGPR-41 & + & + & - & + & + & + & - & + & + & + & + & + & - & + & $3+$ & $3+$ & $4+$ & Azotobacter sp. \\
\hline PGPR-43 & + & + & - & - & + & + & + & + & - & + & + & + & - & + & $4+$ & $3+$ & $4+$ & Azotobacter sp. \\
\hline PGPR-45 & + & + & - & + & + & + & + & + & + & + & + & + & - & + & $3+$ & $2+$ & $3+$ & Azotobacter sp. \\
\hline PGPR-46 & + & + & - & + & + & + & - & + & + & + & + & + & - & + & $3+$ & $2+$ & $4+$ & Azotobacter sp. \\
\hline PGPR-49 & + & + & - & - & + & + & + & + & - & + & + & + & - & + & $4+$ & $3+$ & $4+$ & - \\
\hline PGPR-51 & + & + & - & - & + & + & + & + & - & + & + & + & - & + & $3+$ & $2+$ & $4+$ & Azotobacter sp. \\
\hline PGPR-53 & + & + & - & + & + & + & + & + & + & + & + & + & - & + & $3+$ & $2+$ & $3+$ & Azotobacter sp. \\
\hline PGPR-55 & + & + & - & + & + & + & - & + & + & + & + & + & - & + & $3+$ & $2+$ & $3+$ & Azotobacter sp. \\
\hline PGPR-59 & + & + & - & + & + & + & - & + & + & + & + & + & - & + & $3+$ & $3+$ & $3+$ & Azotobacter sp. \\
\hline PGPR-61 & + & + & - & - & + & + & + & + & - & + & + & + & - & + & $4+$ & $2+$ & $4+$ & Azotobacter sp. \\
\hline PGPR-64 & + & + & - & + & + & + & - & + & + & + & + & + & - & + & $3+$ & $2+$ & $3+$ & Azotobacter sp. \\
\hline PGPR-65 & + & + & - & - & + & + & + & + & - & + & + & + & - & + & $4+$ & $3+$ & $4+$ & Azotobacter sp. \\
\hline PGPR-68 & + & + & - & + & + & + & - & + & + & + & + & + & - & + & $3+$ & $2+$ & $3+$ & Azotobacter sp. \\
\hline
\end{tabular}


Table.3 Morphological and Biochemical characteristics of the Phosphate Solubilizing Bacteria (PSB)

\begin{tabular}{|c|c|c|c|c|c|c|c|c|c|c|c|c|c|c|c|c|c|c|c|}
\hline \multirow{2}{*}{ Isolate } & \multirow{2}{*}{$\begin{array}{l}\text { Spore } \\
\text { forma } \\
\text { tion }\end{array}$} & \multicolumn{17}{|c|}{ Biochemical tests } & \multirow[t]{2}{*}{ Probable genera } \\
\hline & & 1 & 2 & 3 & 4 & 5 & 6 & 7 & 8 & 9 & 10 & 11 & $12 a$ & $12 b$ & 13 & $14 a$ & $14 \mathrm{~b}$ & $14 c$ & \\
\hline PGPR-1 & + & - & - & + & + & + & - & + & + & - & + & - & + & - & + & + & + & - & Bacillus spp. \\
\hline PGPR-3 & + & - & + & - & - & + & - & + & + & - & + & - & + & - & + & + & + & - & Bacillus spp. \\
\hline PGPR-7 & - & - & - & - & + & + & + & - & - & - & + & + & + & - & + & + & + & - & Pseudomonas sp. \\
\hline PGPR-9 & + & - & + & - & - & + & - & + & + & - & + & - & + & - & + & + & + & - & Bacillus spp. \\
\hline PGPR-13 & + & - & - & + & + & + & - & + & - & - & + & - & + & - & + & + & + & - & Bacillus spp. \\
\hline PGPR-19 & + & - & - & + & + & + & - & + & + & - & + & - & + & - & + & + & + & - & Bacillus spp. \\
\hline PGPR-23 & + & - & + & - & - & + & - & + & + & - & + & - & + & - & + & + & + & - & - \\
\hline PGPR-27 & + & - & + & - & - & + & - & + & + & - & + & - & + & - & + & + & + & - & Bacillus spp. \\
\hline PGPR-29 & + & - & - & + & + & + & - & + & + & - & + & - & + & - & + & + & + & - & Bacillus spp. \\
\hline PGPR-33 & - & - & - & - & + & + & + & - & - & - & + & + & + & - & + & + & + & - & Pseudomonas sp. \\
\hline PGPR-37 & + & - & + & - & - & + & - & + & + & - & + & - & + & - & + & + & + & - & Bacillus spp. \\
\hline PGPR-42 & - & - & - & - & + & + & + & - & - & - & + & + & + & - & + & + & + & - & Pseudomonas sp. \\
\hline PGPR-47 & + & - & - & + & + & + & - & + & + & - & + & - & + & - & + & + & + & - & Bacillus spp. \\
\hline PGPR-48 & + & - & + & - & - & + & - & + & + & - & + & - & + & - & + & + & + & - & - \\
\hline PGPR-54 & + & - & + & - & - & + & - & + & + & - & + & - & + & - & + & + & + & - & Bacillus spp. \\
\hline PGPR-57 & - & - & - & - & + & + & + & - & - & - & + & + & + & - & + & + & + & - & Pseudomonas sp. \\
\hline PGPR-66 & + & - & - & + & + & + & - & + & - & - & + & - & + & - & + & + & + & - & Bacillus spp. \\
\hline \multicolumn{5}{|c|}{ 1.Indole production test, } & \multicolumn{6}{|c|}{ 6. Oxidase test } & \multicolumn{6}{|c|}{ 11. Ammonium production test } & \multicolumn{3}{|c|}{ 14a. Glucose } \\
\hline \multicolumn{5}{|c|}{ 2. Methyl red test, } & \multicolumn{6}{|c|}{ 7. Starch hydrolysis test } & \multicolumn{6}{|c|}{ 12a. Acid production } & \multicolumn{3}{|c|}{ 14b. Sucrose } \\
\hline \multicolumn{5}{|c|}{ 3.VogerProskauer test } & \multicolumn{6}{|c|}{ 8. Gelatin liquefaction test } & \multicolumn{6}{|c|}{ 12b. Gas production } & \multicolumn{3}{|c|}{ 14c. Mannitol } \\
\hline \multicolumn{5}{|c|}{ 4. Citrate utilization test } & \multicolumn{6}{|c|}{ 9. $\mathrm{H}_{2} \mathrm{~S}$ production } & & \multicolumn{3}{|c|}{+ : Positive } \\
\hline \multicolumn{5}{|c|}{ 5. Catalase test } & \multicolumn{6}{|c|}{ 10. Motility test } & \multicolumn{6}{|c|}{ 13. Casein hydrolysis } & \multicolumn{3}{|c|}{ - : Negative } \\
\hline \multicolumn{5}{|c|}{ + : Very less growth } & \multicolumn{6}{|c|}{ 2+ : Less growth } & \multicolumn{6}{|c|}{ 3+ : Medium growth } & $4+:$ & lood gi & wth \\
\hline
\end{tabular}


Table.4 Screening of efficient free living nitrogen fixing isolates

\begin{tabular}{|c|c|c|c|}
\hline Sl. No & Isolate code & mg $\mathbf{N}$ per g of $\mathbf{C}$ utilized & IAA $(\mu \mathrm{g}$ per $\mathrm{ml}$ of medium $)$ \\
\hline 1 & PGPR-4 & 7.57 & 12.75 \\
\hline 2 & PGPR-5 & 5.32 & 9.46 \\
\hline 3 & PGPR-8 & 4.81 & 5.61 \\
\hline 4 & PGPR-10 & 7.33 & 11.68 \\
\hline 5 & PGPR-12 & 7.45 & 15.78 \\
\hline 6 & PGPR-17 & 5.13 & 7.78 \\
\hline 7 & PGPR-18 & 9.26 & 18.96 \\
\hline 8 & PGPR-21 & 4.52 & 6.76 \\
\hline 9 & PGPR-24 & 19.33 & 25.12 \\
\hline 10 & PGPR-28 & 6.24 & 12.73 \\
\hline 11 & PGPR-30 & 5.89 & 9.61 \\
\hline 12 & PGPR-31 & 8.56 & 13.52 \\
\hline 13 & PGPR-34 & 14.62 & 19.12 \\
\hline 14 & PGPR-38 & 4.71 & 7.45 \\
\hline 15 & PGPR-41 & 13.22 & 14.52 \\
\hline 16 & PGPR-43 & 15.81 & 17.55 \\
\hline 17 & PGPR-45 & 17.78 & 22.67 \\
\hline 18 & PGPR-46 & 13.59 & 15.32 \\
\hline 19 & PGPR-49 & 4.68 & 5.98 \\
\hline 20 & PGPR-51 & 5.67 & 9.42 \\
\hline 21 & PGPR-53 & 15.12 & 18.84 \\
\hline 22 & PGPR-55 & 6.23 & 12.45 \\
\hline 23 & PGPR-59 & 4.67 & 9.56 \\
\hline 24 & PGPR-61 & 15.54 & 12.53 \\
\hline 25 & PGPR-64 & 17.23 & 19.78 \\
\hline 26 & PGPR-65 & 7.48 & 23.54 \\
\hline 27 & PGPR-68 & & 14.56 \\
\hline \multicolumn{2}{|c|}{ Reference strain (A. chroococcum) } & 18.16 & 24.08 \\
\hline
\end{tabular}

Table.5 Screening of efficient phosphate solubilizing bacterial isolates

\begin{tabular}{|c|c|c|c|c|c|}
\hline \multirow[t]{2}{*}{ SI. No } & \multirow[t]{2}{*}{ Isolate code } & \multicolumn{2}{|c|}{ Per cent $P i$ released } & \multirow{2}{*}{$\begin{array}{c}\text { Phosphate Solubilization } \\
\text { efficiency }(\%)\end{array}$} & \multirow{2}{*}{$\begin{array}{c}\text { IAA Production }(\mu \mathrm{g} \text { per } \mathrm{ml} \text { of } \\
\text { medium) }\end{array}$} \\
\hline & & $5^{\text {th }}$ day & $10^{\text {th }}$ day & & \\
\hline 1 & PGPR-1 & 6.12 & 12.86 & 132.6 & 5.89 \\
\hline 2 & PGPR-3 & 4.80 & 8.32 & 110.4 & 4.54 \\
\hline 3 & PGPR-7 & 5.78 & 10.45 & 114.0 & 10.45 \\
\hline 4 & PGPR-9 & 7.85 & 14.32 & 168.7 & 20.13 \\
\hline 5 & PGPR-13 & 6.23 & 12.32 & 128.0 & 19.95 \\
\hline 6 & PGPR-19 & 5.89 & 10.12 & 115.6 & 15.57 \\
\hline 7 & PGPR-23 & 4.37 & 8.78 & 108.8 & 17.84 \\
\hline 8 & PGPR-27 & 5.36 & 10.33 & 112.6 & 11.48 \\
\hline 9 & PGPR-29 & 4.39 & 8.59 & 109.6 & 6.32 \\
\hline 10 & PGPR-33 & 6.37 & 13.12 & 138.4 & 19.00 \\
\hline 11 & PGPR-37 & 4.40 & 8.89 & 110.0 & 9.92 \\
\hline 12 & PGPR-42 & 3.80 & 7.32 & 102.8 & 15.12 \\
\hline 13 & PGPR-47 & 4.52 & 7.45 & 109.0 & 17.67 \\
\hline 14 & PGPR-48 & 5.78 & 10.91 & 114.8 & 16.48 \\
\hline 15 & PGPR-54 & 5.23 & 9.89 & 106.8 & 18.79 \\
\hline 16 & PGPR-57 & 6.57 & 13.13 & 136.0 & 16.23 \\
\hline 17 & PGPR-62 & 4.35 & 8.56 & 122.8 & 14.99 \\
\hline \multicolumn{2}{|c|}{$\begin{array}{l}\text { Reference strain (Bacillus } \\
\text { magaterium) }\end{array}$} & 7.05 & 13.58 & 150.0 & 19.11 \\
\hline & ntrol & 0.42 & 0.61 & 0.00 & 0.00 \\
\hline
\end{tabular}


Plate.1 Different biochemical tests conducted for identification of PGPR isolates

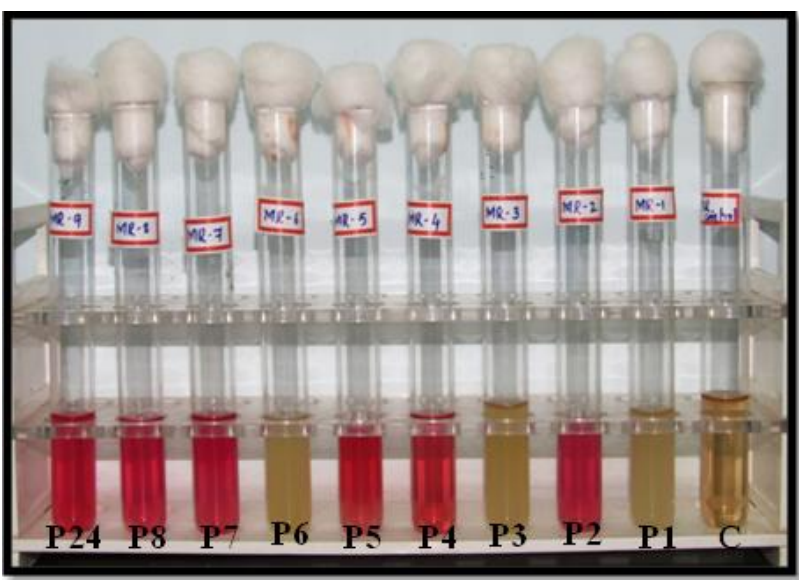

1a. Methyl red test

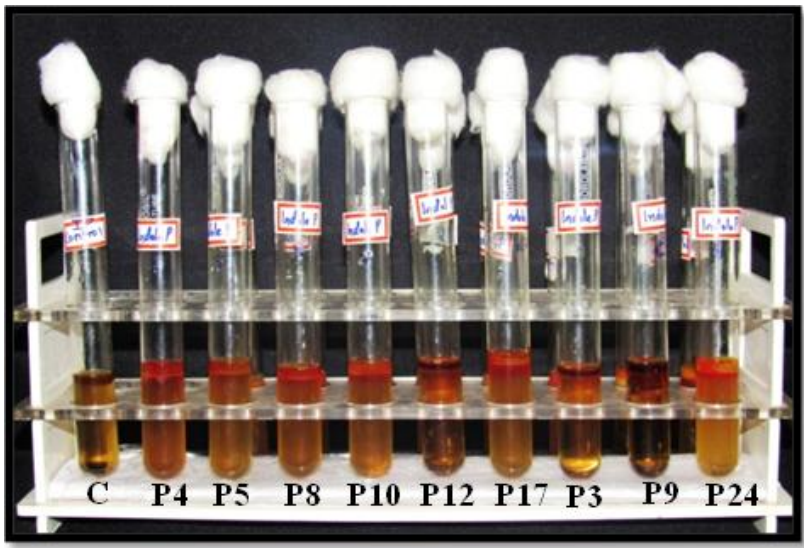

1c. Indole production test

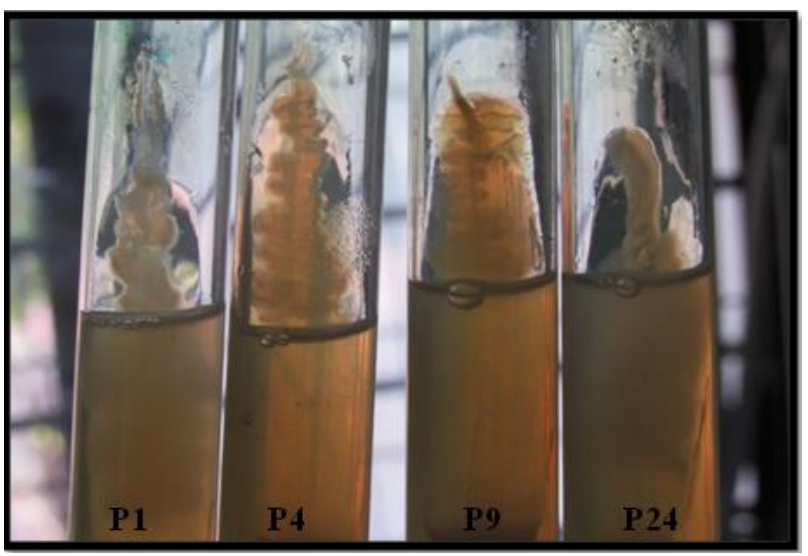

1e. Catalase test

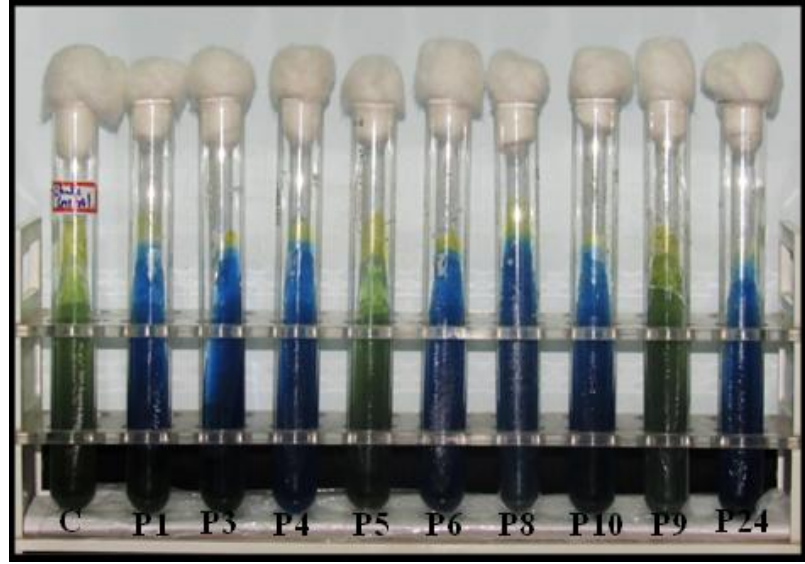

1b. Citrate utilization test

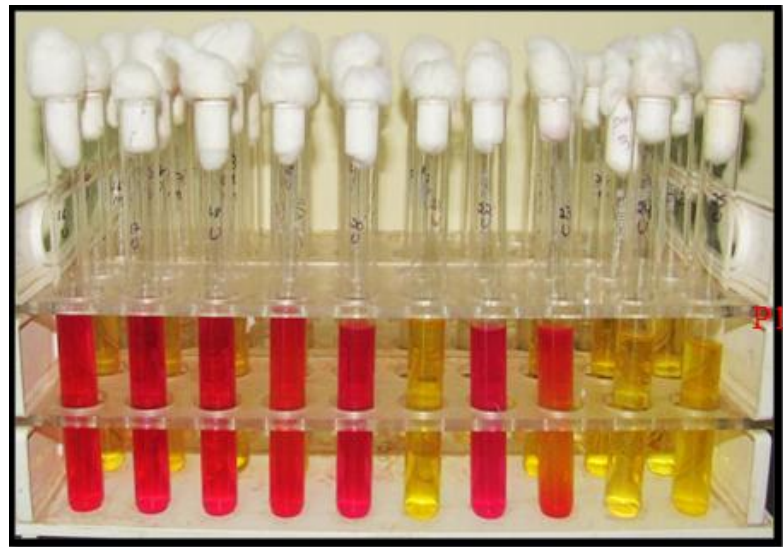

1d. Urease test

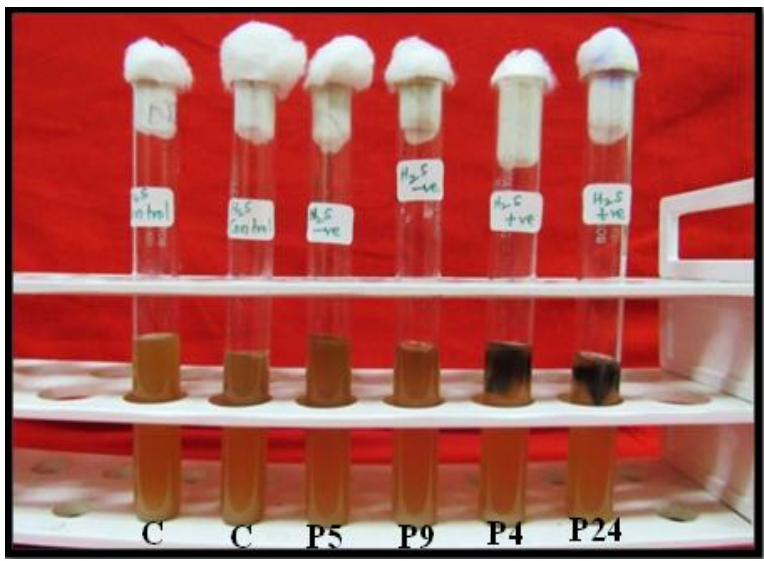

1f. Hydrogen sulphide 


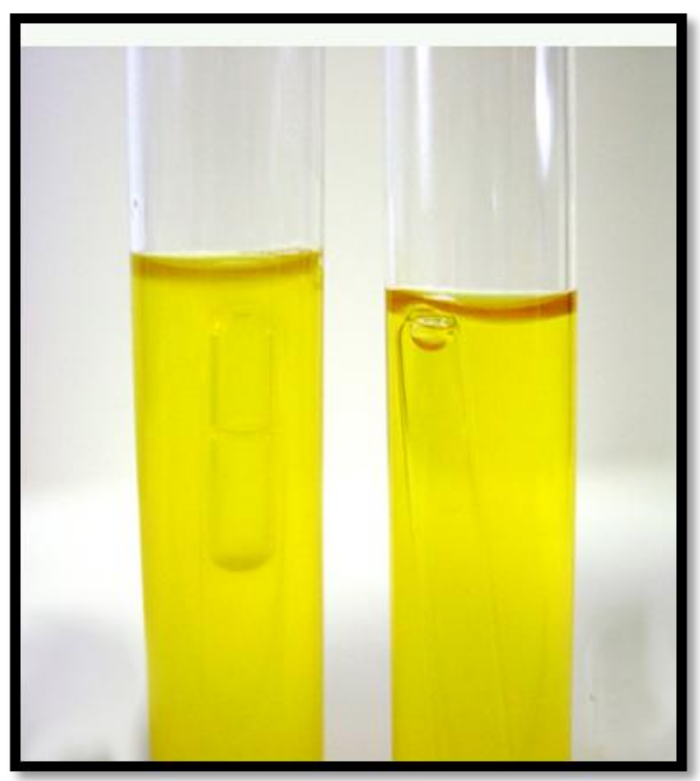

1a. Gas production

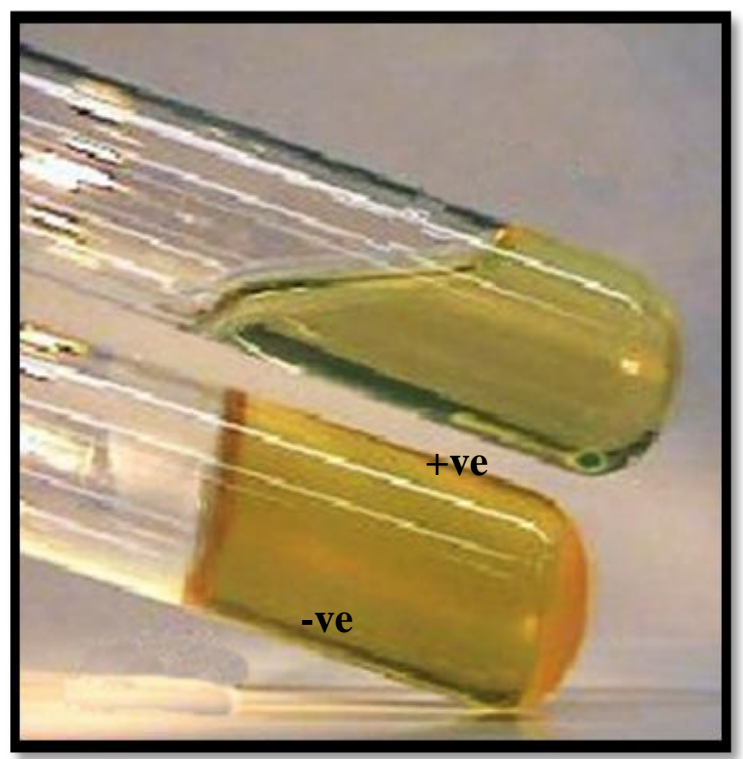

1b. Gelatin

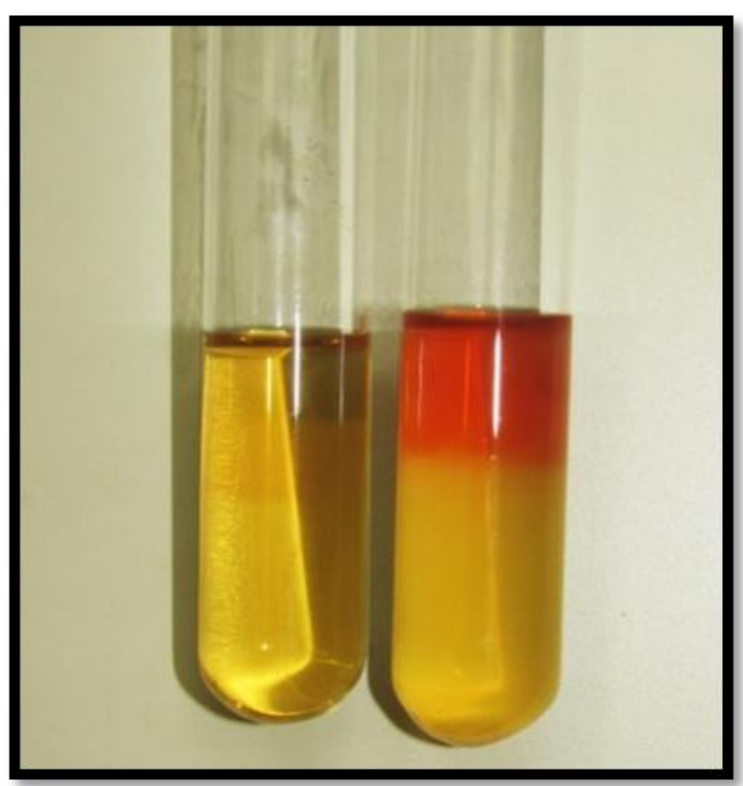

1c. VP test +ve

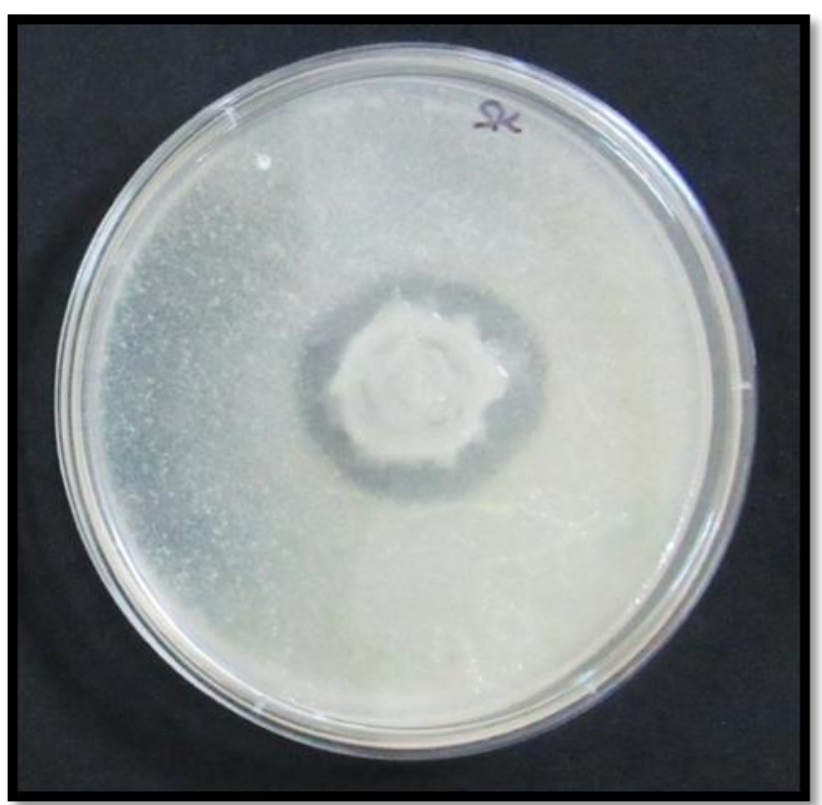

1d. Casein hydrolysis test 
Int.J.Curr.Microbiol.App.Sci (2019) 8(6): 790-803

Plate.2 Cultures of PGPR isolates (Azotobacter sp. and Phosphate solubilizing bacteria)

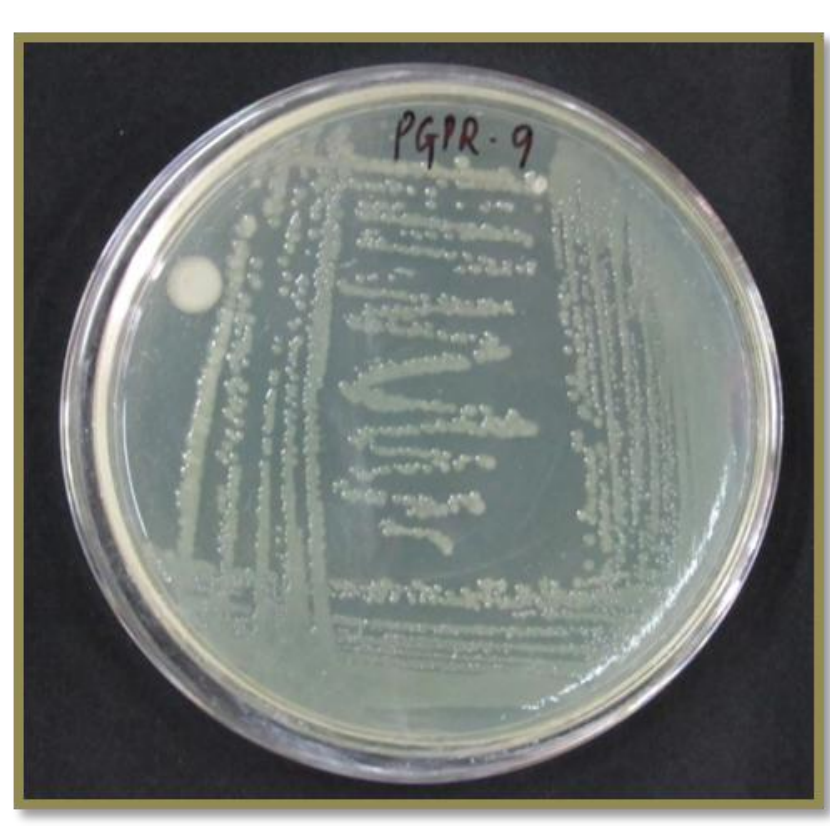

PSB on Nutrient agar media

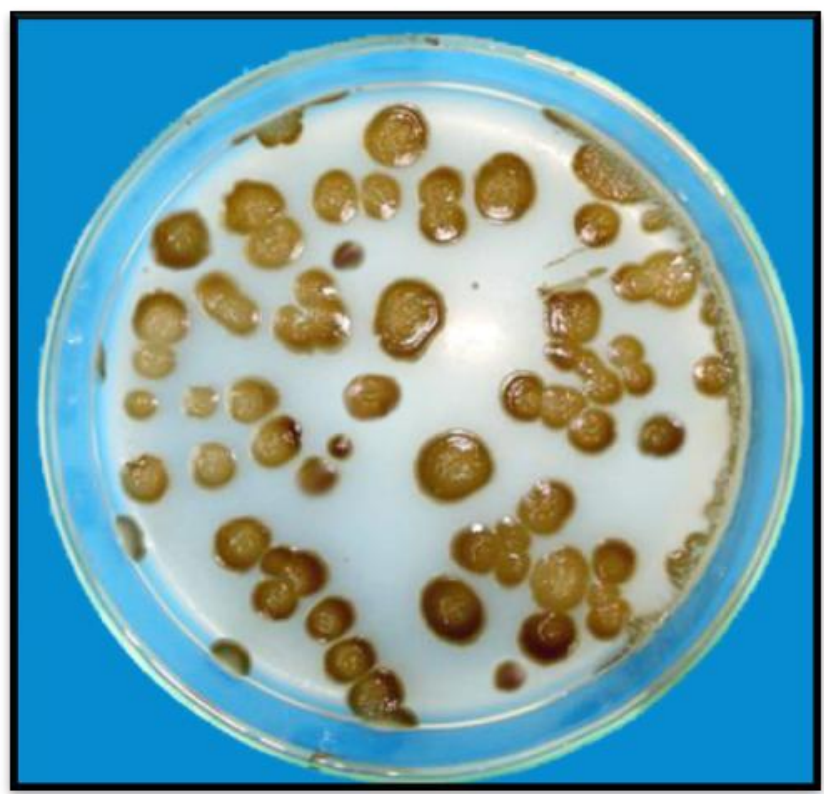

Pigmented Azotobacter sp.

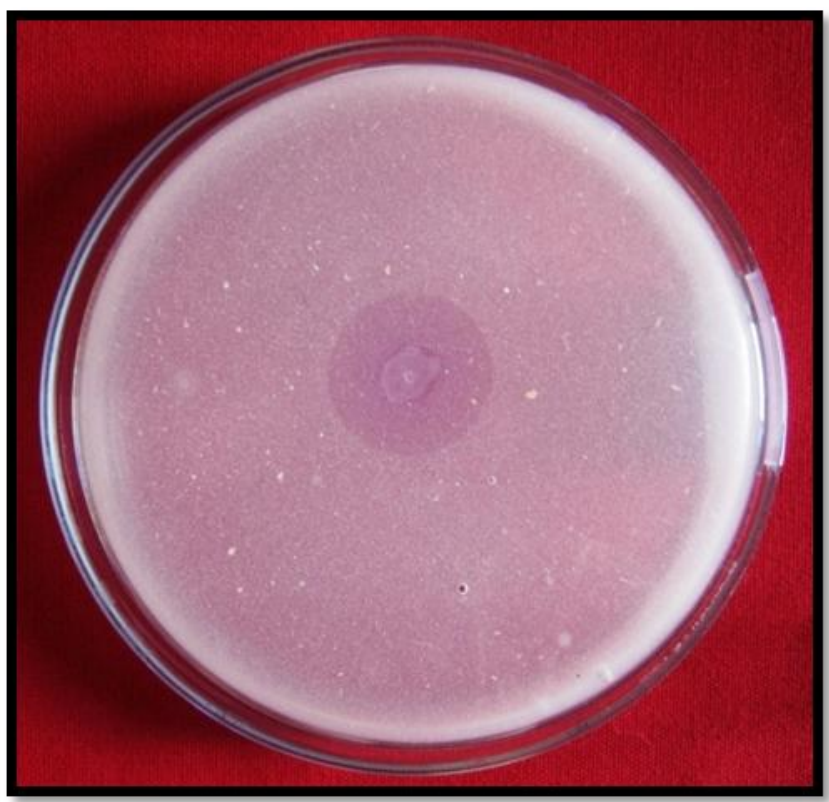

PSB on Pikovskay's media

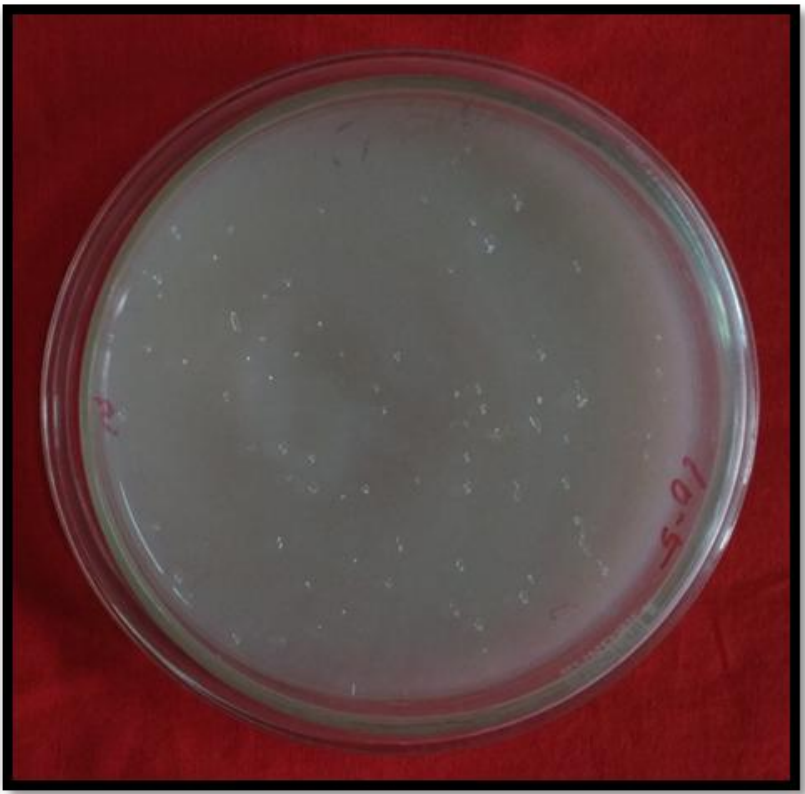

Azotobacter on Whaksman No-77 media 
The lowest Pi released was found in PGPR-62 $(4.35 \%)$ at $5^{\text {th }}$ and $(8.56 \%) 10^{\text {th }}$ day of incubation. Similar observation of solubilization of insoluble phosphate was made by $\mathrm{Hu}$ et al., 2006 and Sen and Paul 1957.

\section{Production of plant growth promoting substances by PGPR isolates}

\section{IAA production by free living $\mathbf{N}_{2}$ fixers}

Higher IAA production was recorded in PGPR-24 $(25.12 \mu \mathrm{g} / \mathrm{ml})$, which is superior over all other isolates. Lowest IAA production $(5.61 \mu \mathrm{g} / \mathrm{ml})$ was recorded in isolate PGPR- 8 details were described in Table 8. The results were on par with the results of Jalan (2003) screened and twelve Azotobacter isolates produce the IAA only 2.09 to $3.3 \mu \mathrm{g}$ per $\mathrm{ml}$.

\section{IAA production by the phosphate solubilizing bacteria}

The higher IAA production was recorded inPGPR-9 $(20.13 \mu \mathrm{g} / \mathrm{ml})$ is superior over all the isolates followed by PGPR-13 (19.95 $\mu \mathrm{g} / \mathrm{ml})$. Lowest production of IAA was in isolate PGPR-3 $(4.54 \mu \mathrm{g} / \mathrm{ml})$. Result is presented in the Table 9. Geeta (2001) studied twenty eight P-solubilizing bacterial strains for the production of PGPS. The production of IAA among the strains varied from 3.61$35.45 \mathrm{~g} / 25 \mathrm{ml}$ of broth. Similar results were found by Barea et al., (1976) and Leinhos and Vacek (1994).

In conclusion, in the present scenario use of microorganisms in the field of agriculture to enhance growth, yield and quality of crop is the best way to reduce cost of production and maintain ecological harmony. Rhizosphere is rich source of beneficial microbes which have potential to improve plant growth.

\section{References}

Antoun, H. And Kloepper, J., 2001, Plant growth promoting rhizobacteria. In: Encyclopedia of Genetics, (Eds. Brenner, S. and Miller, J.), Academic Press, pp.1477-1480.

Barea, J. M., Navamo. E. and Monotoya, E., 1976, Production of plant growth regulators by rhizosphere phosphate solubilizing bacteria. J. Appl. Bacteriol., 40: 129-134.

Cappuccino, J. C. and Sherman, N., 1992, In: Microbiology: A Laboratory Manual, New York, pp. 125-179.

Das, A. J., Kumar, M. and Kumar, R., 2013, Plant growth promoting rhizobacteria (PGPR): An alternative of chemical fertilizer for sustainable, environment friendly agriculture. Res. J. Agric. For. Sci., 1(4): 21-23.

Geeta, S. K., 2001, Analysis of PGPS mutants of MPS bacteria and their effect on plant growth and nutrient uptake. M. Sc. (Agri.) Thesis, Univ. of Agril. Sci., Dharwad.

Glick, B. R., 1995, The enhancement of plant growth by free-living bacteria. Can. J. Microbiol., 41: 109-117.

Goswamy, 1976, Worth of Azotobacter as a bacteriâl fertilizer. Fert. News' 2L' 2128.

Hiltner, L., 1904, Lorenz Hiltner, a pioneer in rhizosphere microbial ecology and soil bacteriology research. Pl. Soil, 312: 714.

Hu, X. F., Chen, J. and Guo, J. F., 2006, Two phosphate and potassium solubilizing bacterial isolated from Tiannu Mountain, Zhijiang, china. World J. Microbiol. Biotehcnol., 22: 983-990.

Ivanova, E. G., Doronina, N. V. and Trotsenko, Y. A., 2001, Aerobic methylobacteria are capable of synthesizing auxins. Microbiol., 70: 345-347. 
Jackson, M. L., 1973, Soil Chemical Analysis, Prentice Hall of India Pvt. Ltd., New Delhi.

Jalan., 2003, Screening bacteria for plant growth promotion activities in vitro. Indian J. Agri. Sci. 4(1): 27-31.

Kanimozhi, G. and Panneerselvam, A., 2010, Isolation and Screening of phosphate solubilising fungi from Mangrove soils of Muthupettai, Thiruvarur District, India. Asian J. Microbiol. Biotechnol. Env. Sci., 12(2): 359-361.

Kizilkaya, R., 2009, Nitrogen fixation capacity of Azotobacter spp. strains isolated from soils in different ecosystems and relationship between them and the microbiological properties of soils. J. Environ. Biol., 30(1): 78-82.

Leinhos, V. and Vacek, O., 1994, Biosynthesis of auxins by phosphate solubilizing rhizobacteria from wheat and rye. Microbiol. Res., 149: 31-35.

Pikovskaya, R. I., 1948, Mobilization of phosphorus in soil in connection with vital activity of some microbial species. Microbiol., 17: 362-370.

Schroth, M. N. and Hancock, J. G., 1982, Disease-suppressive soil and rootcolonizing bacteria. Sci., 16: 1376-1381.

Sharma, K., Dak, G., Agarwal, A., Bhatnagar, M. and Sharmar, 2007, Effect of phosphate solubilizing bacteria on the germination of Cicer arietinum seeds and seedling growth. J. Herb Med. Toxicol., 1(1): 61-63.

Vijaypal, I., Jalalipal, V. and Jalali, I., 1998, Rhizosphere bacteria for biocontrol of plant diseases. Ind. J. Microbiol., 38: 187-204.

\section{How to cite this article:}

Praveen Ranadev, K. Nagaraju, R. Muthuraju and Vasantha Kumari, R. 2019. Studies on Isolation, Characterization and in-vitro Screening of Plant Growth Promoting Rhizobacteria from Rhizospheric Soil of Chrysanthemum (Dendranthema grandiflora Tzvelev.). Int.J.Curr.Microbiol.App.Sci. 8(06): 790-803. doi: https://doi.org/10.20546/ijcmas.2019.806.096 